Courrier du Centre international Blaise Pascal

$16 \mid 1994$

Varia

\title{
De la $\mathrm{XI}^{\mathrm{e}}$ Provinciale aux Pensées
}

\section{Dominique Descotes}

\section{OpenEdition \\ Journals}

Édition électronique

URL : http://journals.openedition.org/ccibp/593

DOI : 10.4000/ccibp.593

ISSN : 2493-7460

\section{Éditeur}

Centre international Blaise Pascal

\section{Édition imprimée}

Date de publication : 5 avril 1994

Pagination : $35-38$

ISSN : 0249-6674

\section{Référence électronique}

Dominique Descotes, «De la XleProvinciale aux Pensées », Courrier du Centre international Blaise Pascal [En ligne], 16| 1994, mis en ligne le 07 janvier 2016, consulté le 20 avril 2019. URL : http:// journals.openedition.org/ccibp/593 ; DOI : 10.4000/ccibp.593

Ce document a été généré automatiquement le 20 avril 2019.

Centre international Blaise Pascal 


\title{
De la $\mathrm{XI}^{\mathrm{e}}$ Provinciale aux Pensées
}

\author{
Dominique Descotes
}

1 Le problème de la raillerie chrétienne occupe dans l'œuvre de Pascal une place dont la XI Provinciale mesure l'importance: Pascal a cru devoir se justifier sur le style de ses premières lettres, et malgré cette justification, il a opté ensuite pour une rhétorique plus sévère : signe qu'il s'est senti atteint en un point sensible. Il s'agit en fait là d'un aspect particulier d'un problème auquel s'est plusieurs fois heurté le parti de l'action à Port-Royal, celui de la violence rhétorique dans les disputes religieuses. Arnauld lui a consacré plusieurs opuscules : la Réponse à la lettre d'une personne de condition (1654) a inspiré la XI Provinciale ; l'un des chapitres du Renversement de la Morale de Jésus-Christ par les Calvinistes (1672) a été publié dans un recueil de lettres sous le titre de Réponse à la plainte que l'on fait à M. Arnauld des termes injurieux dont il se sert pour décrier la morale de ses adversaires; en 1679, la Dissertation selon la méthode des géomètres pour la justification de ceux qui emploient en écrivant dans certaines rencontres des termes que le monde estime durs fait écho aux disputes suscitées par les attaques de Mallet contre le Nouveau Testament de Mons ${ }^{1}$. Nicole a traité le même sujet dans les notes de Wendrock et les Imaginaires. En général, ce sont moins à leurs ennemis déclarés qu'ils répondent qu'à des amis mécontents de leur vigueur polémique, qui plaident en faveur d'un style plus doux.

2 Pour ce qui touche les Pensées, Voltaire n'a comme toujours pas manqué l'objection : il les rattache directement aux Provinciales en ce que Pascal y écrit « contre la nature humaine à peu près comme il écrivait contre les jésuites ", en disant « éloquemment des injures au genre humain ${ }^{2}$ ». Le reproche touche au fond même du livre. Les fragments 188 et 194 attestent que Pascal s'est bien posé le problème du droit de l'apologiste d'user d'invective. Mais si vraiment les Pensées calomnient l'homme, n'est-on pas en droit de rétorquer à Pascal la péroraison de sa XII Provinciale : «Les injures que vous me dites n'éclairciront pas nos différends, et les menaces que vous me faites en tant de façons ne m'empêcheront pas de me défendre »?

3 "J'appelle réfuter avec douceur», dit Arnauld, "quand on a pour but en réfutant quelqu'un, de l'épargner autant que l'on peut, sans trahir la cause de la vérité ; d'excuser ses fautes, de couvrir ses défauts et de ne porter aucune atteinte à sa réputation ». « Réfuter fortement », c'est " quand on a pour but (l'intérêt de la vérité et de l'Église le 
demandant ainsi) d'humilier et de confondre un écrivain emporté, qui emploie toute sorte de méchants moyens pour décrier la vérité [...], ce qui oblige, afin de le faire connaître pour tel qu'il est, de ne point dissimuler ses excès en les représentant fortement, mais sans exagérer au-delà de ce qui en est ${ }^{3} »$. La différence n'est pas seulement dans l'emploi des «termes durs» comme «calomnie, mensonge, impertinence ", elle tient surtout dans une mise en cause directe des personnes qui rend la réfutation plus blessante pour l'amour-propre. Il va de soi que la réfutation dure a pour fin non seulement de rappeler l'adversaire à la vérité, mais aussi de prévenir contre lui les personnes de bonne volonté s'il s'obstine dans l'erreur.

Pascal n'aime guère l'éloquence «qui persuade par douceur, non par empire, en tyran non en roi $^{4}{ }^{»}$. Les adoucissements ne sont pas son affaire. Les termes durs abondent dans les Pensées : misère, folie, faiblesse, vanité, sottise, ridicule, autant de traits qui frappent tour à tour les incrédules, les philosophes, les demi-habiles et alii. Quant à la mise en cause des personnes, elle est partout : l'apologiste dénonce sans ménagement les vices de Zénon, les défauts de Montaigne, l'ambition scientifique de Descartes, la paresse de Mitton, les impostures de Mahomet. Ce sont là des individualités typiques, mais on mesure la force de la réfutation aux réactions qu'elle provoque aujourd'hui encore parmi les épigones plus ou moins lointains de ces grandes figures du théâtre philosophique. S'agit-il pour autant d'une rhétorique violente? La réfutation forte, dit Arnauld, respecte l'adéquation des fins et des moyens: "Quand on s'est proposé une fin bonne, louable, importante ", comme rappeler à la vérité ceux que l'amour-propre égare et mettre en garde ceux qu'ils pourraient tromper, «on fait bien de choisir, entre les moyens légitimes, ceux qui sont les plus propres pour arriver à cette fin »; et si l'on n'use que du vrai et d'expressions adéquates, des termes durs peuvent être « un moyen non seulement légitime, mais nécessaire ${ }^{5}$ ». La réfutation violente au contraire emploie des moyens tyranniques qui déshonorent la vérité. C'est pour les discerner que la $\mathrm{XI}^{\mathrm{e}}$ Provinciale propose plusieurs règles pour juger «si les répréhensions partent d'un esprit de piété et de charité, ou d'un esprit d'impiété et de haine ». La question est donc de savoir si Pascal les respecte dans les Pensées.

5 La première est si évidente qu'elle est sous-entendue dans la $\mathrm{XI}^{e}$ Provinciale : la réfutation forte n'est légitime que dans une situation où la défense de la vérité est nécessaire et urgente. Arnauld pose par exemple en axiome qu'il faut que la vérité ait contre elle de grands préjugés dans l'esprit du public, surtout s'ils reposent sur « des ignorances et des faussetés ${ }^{6}$ ». Tel est bien, d'après les Pensées, le cas de la religion chrétienne: «Les hommes ont mépris pour la religion, ils en ont haine et peur qu'elle soit vraie »; et quoique nul ne doute " qu'il ne faille exposer sa vie pour défendre le bien public », on ne le fait pas "pour la religion ${ }^{7}$ ». Pour la gravité de la situation, la $\mathrm{XV}^{\mathrm{e}}$ Provinciale par exemple justifie la dénonciation des calomniateurs par la nécessité de défendre les innocents et d'éclairer les fidèles sur les méthodes des jésuites. Mais les Pensées sont plus pressantes encore : s'il n'y a pas urgence à approfondir une "question de philosophie » comme "l'opinion de Copernic », « il importe à toute la vie de savoir si l'âme est mortelle ou immortelle ", et le plus tôt possible puisqu'il est incertain si on sera une heure au monde ${ }^{8}$. La réfutation doit être d'autant plus forte que les incroyants résistent aux appels les plus insistants pour courir à l'aveuglette « dans le précipice ». Ce cas paraît différer de celui de la XVe Provinciale, qui justifie la vigueur de la rhétorique par la nécessité de neutraliser des personnes nuisibles à autrui comme sont les casuistes; mais ce n'est qu'une variante où nuisance et victime coïncident, les incrédules qui font «le brave 
contre Dieu » pour paraitre avoir "secoué le joug » se font du mal à eux-mêmes. Mais si l'on veut un argument semblable en tout point, il faut le chercher dans les traits du fragment 644 contre les apologistes maladroits: pourquoi Pascal s'en prend-il si vertement aux auteurs qui tirent leurs preuves de Dieu du «cours de la lune et des planètes ", sinon parce qu'ils déçoivent et détournent de la religion des lecteurs qui cherchent sincèrement? Ce genre d'allié néfaste mérite bien qu'on le renvoie fermement à la niche.

6 La première règle de la $\mathrm{XI}^{\mathrm{e}}$ Provinciale est «que l'esprit de piété porte toujours à parler avec vérité et sincérité; au lieu que l'envie et la haine emploient le mensonge et la calomnie »: "vera dicere, et cum causa ", dit Arnauld. Ce principe simple en apparence enferme trois problèmes distincts: l'un, d'ordre psychologique, touche la sincérité de l'auteur; le second concerne l'exactitude des faits allégués, et le troisième, d'ordre rhétorique, la justesse des termes employés. Comme on peut bien être sincère et se tromper ou employer un mot mal à propos, chacun demande un examen à part.

7 Par nature, la question de la sincérité est insoluble. On peut même se demander si elle a un sens, l'apologiste des Pensées étant une pure création littéraire. Cependant en plusieurs passages, Pascal a semé des traits qui sont autant de marques de la sincérité de l'auteur. À l'expression du fragment 681, «en vérité je leur dirais ce que j'ai dit souvent, que cette négligence n'est pas supportable », Voltaire répond: «À quoi bon nous apprendre que vous l'avez dit souvent? » C'est qu'une pensée répétée souvent compte d'ordinaire parmi celles dont on est profondément persuadé : l'auteur semble donc exprimer le fond de sa pensée. De même lorsqu'il affiche envers les incrédules une compassion qui ne doit rien à son intérêt propre, mais qu'inspire l'intérêt d'autrui, le lecteur applique inconsciemment le principe juridique qu'on peut faire confiance à un homme lorsqu'aucun motif d'amourpropre ne vient déranger l'ordre naturel de son comportement ${ }^{9}$.

8 Le problème de la vérité est résolu par l'appel à l'expérience personnelle du lecteur, auquel Pascal laisse juger si telle conduite humaine comporte ou non de la vanité, de la faiblesse ou de l'impertinence. De la faiblesse : chacun sait à quel point la réflexion la plus importante peut être arrêtée par le bourdonnement d'une mouche. De l'inconstance: « un homme vit avec plaisir en son ménage. Qu'il voie une femme qui lui plaise, qu'il joue cinq ou six jours avec plaisir, le voilà misérable s'il retourne à sa première occupation. Rien n'est plus ordinaire que cela ». L'observation de soi au moment même de la lecture des Pensées apporte aussi des preuves sur le vif: "Qu'une chose aussi visible qu'est la vanité du monde soit si peu connue, que ce soit une chose étrange et surprenante de dire », par exemple au lecteur de l'Apologie, "que c'est une sottise de chercher les grandeurs, cela est admirable». Chacun constate ainsi sur lui-même la justesse des reproches de Pascal ${ }^{10}$.

9 Troisième aspect : la justesse des mots dans leur dureté même. « Le moyen le plus propre pour arriver à la fin [...] est de nommer les choses par leurs noms, et appeler impertinence ce qui est impertinence »; « il est permis et souvent même nécessaire dans les disputes de religion, d'appeler erreur ce qui est erreur, impie ce qui est impie, extravagant et ridicule ce qui l'est en effet $^{11}{ }^{1}$. Le P. Garasse a tort de reprocher à Charron d'avoir « la tête pleine d'écrevisses »; mais il n'est pas inadéquat de taxer de vide et de sottise les stoïciens qui, après avoir promis le bonheur, proposent le suicide, ou Mahomet dont le paradis est grossièrement épicurien, ou encore les philosophes qui recommandent aux hommes de chercher leur bien en eux-mêmes, alors qu'il est d'expérience courante que "nous sommes pleins de choses qui nous jettent au-dehors", et que, dès qu'on 
s'isole, le sentiment de la misère et l'ennui emplissent l'esprit de leur venin. Dans les Pensées, Pascal emploie généreusement l'adjectif ridicule pour qualifier ces maîtres à penser que leurs bourdes suffisent à discréditer une fois pour toutes : se moquer de ces frères du P. Noël n'est pas autre chose qu'appeler un chat un chat.

Cette première règle est modérée par le devoir de discrétion : «Ce n'est pas assez [...] de ne dire que des choses vraies, il faut encore ne pas dire toutes celles qui sont vraies, parce qu'on ne doit rapporter que les choses qu'il est utile de découvrir, et non pas celles qui ne pourraient que blesser sans porter aucun fruit. " Pascal en use ainsi à l'égard des libertins. Il ne retient contre eux que les motifs qui touchent à l'essentiel: ils ne se soucient pas de la vérité, ils ont complètement perdu le sens de leur intérêt propre et de leur salut, ils suivent les voies qui les conduisent tout droit vers leur perte. Même lorsqu'il met en cause leurs passions, il ne les présente qu'en termes généraux, comme obstacles à la recherche, et sans entrer dans le détail. Mais d'autres ne se privent pas d'employer une artillerie nettement plus ravageuse. Que les libertins sont victimes d'une " humeur mélancolique » qui les porte au "mépris de toutes choses», qu'ils ont « des esprits traversiers, contrefaits et bizarres ", mis " au monde pour faire rire les hommes ", voilà les premières douceurs que leur adresse le jésuite Garasse. Sur les débordements moraux qui engendrent l'incroyance, il est aussi beaucoup plus précis : les libertins ont pour devise "ambulare per tabernas, volitare per popinas", ils "voltigent parmi les cabarets ", ne connaissent "d'autre Dieu que leur ventre ", vivent dans des abîmes de gourmandise et d'ivrognerie. On connaît l'un d'eux pour être « ivre comme une pie et soûl jusques au sifflet, après mille vilainies qui feraient honte aux cannibales ». Et "puisque les Dames s'en mêlent, je ne sais qu'en dire ${ }^{12}$ ». On sait bien que le P. Garasse a un style très personnel, mais le fragment 681 est d'une tout autre veine.

11 La même discrétion paraît dans la réfutation de l'Islam dans «Fausseté des autres religions ». Là aussi les griefs touchent le fond du sujet, c'est-à-dire l'autorité de Mahomet comme fondateur de religion. À la différence du Christ, Mahomet se dit prophète, mais comme n'importe qui peut en dire autant de soi, comme il n'a pas été prédit et n'a aucun "témoin ", il n'a nulle autorité. Sa religion s'est établie par des moyens purement politiques, la violence et le Djihad : il «a pris la voie de réussir humainement », alors que le Christ «a pris celle de périr humainement» et de faire "tuer les siens", de sorte que l'expansion du christianisme tient visiblement du miracle. D'autre part l'Islam est intrinsèquement tyrannique car Mahomet défend de lire les textes sacrés, et cherche à asservir les esprits dans l'obscurantisme, alors que le Christ et les apôtres ordonnent de lire l'Écriture. Enfin l'Alcoran est ridicule en toutes ses parties, contrairement à la Bible qui, à côté de passages bizarres, contient «des clartés admirables et des prophéties manifestes et accomplies ». Si discutables que puissent être ces reproches, inspirés en général par le De Veritate Religionis christianae de Grotius, ils témoignent de la volonté pascalienne de se tenir à l'essentiel dans le grand débat religieux qui oppose le Christianisme à l'Islam. Mais Grotius et certains libertins lui fournissaient bien d'autres indications, et des plus croustillantes, propres à discréditer le Prophète : que Mahomet était " projectus ad libidinem per omniam vitam », qu'il faisait passer ses crises d'épilepsie pour des extases et des signes de Dieu, qu'il se faisait prédire par un astrologue stipendié pour lui frayer le chemin, qu'il présentait comme l'ange Gabriel un pigeon blanc qui lui picorait des grains de blé sur l'oreille, qu'il avait placé un sbire au fond d'un puits pour se faire proclamer «bien-aimé de Dieu » par les entrailles de la Terre, quitte à faire ensuite combler le puits sur son complice pour éviter tout bavardage gênant. Rien non plus dans 
les Pensées sur certains miracles biscornus comme celui du chameau qui parlait au Prophète, ou de ce bout de Lune qui lui était tombé dans les mains et qu'il remit en place pour rendre à cet astre sa rotondité. Ce n'est certes pas la crainte d'une fatwa qui peut expliquer le silence de Pascal sur ces détails ${ }^{13}$.

La troisième règle oblige à n'employer la raillerie "que contre les erreurs ». Pascal l'applique aux Philosophes, comme on le sait par l'Entretien avec M. de Sacy: il accorde à Platon, aux Stoïciens, aux Pyrrhoniens d'avoir connu une part de la vérité, et ce n'est qu'aux erreurs suscitées par la concupiscence propre à chaque secte qu'il s'attaque. On peut en dire autant des Juifs, dont il blâme le caractère charnel, non sans reconnaître l'existence des spirituels, les "chrétiens de la Loi ancienne». Mutatis mutandis, le fragment 579 résume cette troisième règle: "quand on veut reprendre avec utilité, et montrer à un autre qu'il se trompe, il faut observer par quel côté il envisage la chose, car elle est vraie ordinairement de ce côté-là, et lui avouer cette vérité, mais lui découvrir le côté par où elle est fausse ».

Enfin « le principe et la fin » de toutes ces règles est que « l'esprit de charité porte à avoir dans le cour le désir du salut de ceux contre qui on parle ${ }^{14} »$. Dans les Provinciales, ce précepte ne donne lieu qu'à une application négative, Pascal défiant les Jésuites de prouver qu'il ne le respecte pas. Il acquiert dans les Pensées une tout autre portée. C'est dans cette perspective que Pascal y envisage le problème rhétorique de l'invective: « commencer par plaindre les incrédules. Ils sont assez malheureux par leur condition. Il ne les faudrait injurier qu'au cas que cela servit. Mais cela leur nuit ${ }^{15}$ ». Comme c'est souvent le cas, le jugement de Pascal varie avec le point de vue auquel il se place. Le fragment 300 paraît inspiré par une sorte de désespoir sinistre «ceux qui croient que le bien de l'homme est en sa chair et le mal en ce qui le détourne du plaisir des sens, qu'ils s'en soûlent et qu'ils y meurent». Une note du fragment 662 éclaircit cet étrange anathème : « cela montre qu'il n'y a rien à leur dire : non par mépris, mais parce qu'ils n'ont pas le sens commun. Il faut que Dieu les touche ». Pascal se place ici du point de vue des forces purement humaines de persuasion, et il est bien contraint de convenir que, dans ces limites, toute rhétorique naturelle est impuissante contre certaine forme extrême de l'incroyance : Dieu seul peut briser la prison de concupiscence où se barricade l'athée endurci. Tout au plus peut-on remontrer l'absurdité d'une attitude qui fait du désespoir un sujet de joie : «le beau sujet de se réjouir et de se vanter la tête levée en cette sorte : Donc réjouissons-nous, vivons sans crainte et sans inquiétude et attendons la mort puisque cela est incertain et nous verrons alors ce qu'il arrivera de nous. Je n'en vois pas la conséquence "; " cette négligence en une affaire où il s'agit d'eux-mêmes, de leur éternité, de leur tout, m'irrite plus qu'elle ne m'attendrit ; elle m'étonne et m'épouvante : c'est un monstre pour moi $^{16} »$.

14 Cependant la résignation est intenable dès qu'on se place du point de vue chrétien : la charité oblige à ne pas abandonner les incrédules à leur sort. «Il faut bien être dans la religion qu'ils méprisent pour ne les pas mépriser » jusqu'à «les abandonner dans leur folie. Mais, parce que cette religion nous oblige de les regarder toujours, tant qu'ils seront en cette vie, comme capables de la grâce qui peut les éclairer [...] il faut [...] les appeler à avoir pitié d'eux-mêmes, et à faire au moins quelques pas pour tenter s'ils ne trouveront pas de lumières ${ }^{17}$ ». L'apologiste chrétien, même lorsqu'il connaît ses propres limites et croit la lutte sans espoir, a le devoir irrécusable d'espérer le salut des pécheurs les plus endurcis. 

forces à s'en instruire » et « ceux qui vivent sans s'en mettre en peine et sans y penser » : les premiers méritent une pitié qui "naît de tendresse », au sens où Pascal l'entend: la capacité de partager les intérêts du prochain, éclairée par la charitée ${ }^{18}$. Mais pour «ceux qui en font vanité », il faut invectiver pour les réveiller et les ramener à eux-mêmes. De là les formules les plus sévères des Pensées « rien n'est plus lâche que de faire le brave contre Dieu »; "qu'ils soient au moins honnêtes gens, s'ils ne peuvent être chrétiens "; "ils servent [...] admirablement à montrer la corruption de la nature par des sentiments si dénaturés "; de là enfin le discours ironique prêté à l'athée insouciant qui le tourne en ridicule par un procédé qui rappelle les Provinciales.

Mais l'application de cette dernière règle prend dans les Pensées une signification nouvelle. Dans les Petites Lettres, un fossé infranchissable séparait l'auteur des casuistes corrompus et des Jésuites : même s'il souhaite leur salut, « Montalte » n'a pas grand-chose de commun avec eux, et l'on sent bien qu'ils ne pourront redevenir ses frères qu'au prix d'un reniement complet de leur doctrine morale. Un pas de plus conduirait au pharisaïsme. Le ton des Pensées est tout autre: la religion qu'il défend interdit à l'apologiste de se croire très différent des incrédules contre lesquels il écrit ; elle l'oblige « de croire qu'ils peuvent être dans peu de temps plus remplis de foi que nous ne sommes, et que nous pouvons au contraire tomber dans l'aveuglement où ils sont ", de sorte qu'il «faut faire pour eux ce que nous voudrions qu'on fit pour nous si nous étions à leur place ». La «tendresse » dont le chrétien doit faire preuve envers les athées n'est qu'une autre forme du souci de son propre salut, et les invectives qu'il leur adresse, loin d'être des marques de pharisaïsme, sont la preuve de la claire conscience qu'il doit garder du danger qu'il court à tout instant d'être abandonné de Dieu et destitué de grâce. L'invective ne traduit, dans ces conditions, aucune hostilité.

Loin d'être restreinte à la polémique de 1656, la doctrine de la XI ${ }^{e}$ Provinciale marque donc profondément la technique des Pensées. Pascal s'est visiblement soucié de respecter les règles qu'il avait lui-même posées. Mais il en a aussi approfondi la signification. Si les invectives de la réfutation dure ressemblent aux potions amères que le médecin inflige au malade pour le guérir, en sachant qu'il en aura peut-être besoin lui-même un jour, l'Apologie apparaît à sa manière comme une sorte de méditation sur «le bon usage des maladies ».

\section{NOTES}

1. Arnauld, Euvres, t. XXVII ; la Dissertation se trouve p. 50 sq. Le chapitre X du Livre I du Renversement de la Morale de Jésus-Christ, Desprez, 1672, p. 81 sq. est donné dans le Recueil de plusieurs Lettres de Monsieur Arnauld, Docteur de Sorbonne. Liège, 1698, p. 7 sq. Je cite les Provinciales dans l'édition Cognet, Garnier, 1992.

2. Voltaire, Lettres philosophiques, éd. Naves, Garnier, 1964, p. 141.

3. Arnauld, Dissertation..., loc. cit., p. 50-51.

4. Sel. 584.

Courrier du Centre international Blaise Pascal, 16 | 1994 
5. Arnauld, Dissertation..., p. 51-52.

6. Ibid., p. 52-53.

7. Sel. 46 et 458 .

8. Sel. 183, 195, 196.

9. Domat, Les lois civiles, II, p. 348.

10. Sel. 81, 114, 50.

11. Arnauld, Dissertation..., p. 55 et Réponse à la plainte.... in Recueil..., p. 30.

12. Voir les extraits de Garasse. "La Doctrine curieuse des beaux esprits de ce temps ", 1623, dans Adam. Les libertins au XVII siècle, Buchet-Chastel, 1964, p. 35 sq.

13. Voir Grotius, De Veritate ..., VI, passim, et les extraits de Gabriel Naudé, Considérations politiques..., in Adam, op. cit., p. 143-145.

14. Provinciales, p. 206.

15. Sel. 194.

16. Sel 662 et 681.

17. Sel. 681.

18. Voir le passage de la Vie de Pascal par Gilberte, essentiel sur ce point, in Euvres complètes, I, éd. Mesnard, p. 631-632.

\section{AUTEUR}

\section{DOMINIQUE DESCOTES}

CERHAC, Université Blaise Pascal 